\title{
Schwanoma do Membro Superior: Análise Retrospectiva de 17 Casos
}

\section{Schwannoma of the Upper Extremity: Retrospective Analysis of 17 Cases}

\author{
Ângelo SÁ $\mathbb{1}^{1}$, Leandro Nobre AZEVEDO ${ }^{1}$, Luísa CUNHA ${ }^{1}$ \\ Acta Med Port 2016 Sep;29(9):519-524 - http://dx.doi.org/10.20344/amp.6906
}

RESUMO

Introdução: O schwanoma ou neurilemoma é um tumor benigno dos nervos periféricos e apresenta-se habitualmente como uma lesão única de crescimento indolente, com origem na proliferação das células de Schwann. Representa uma pequena percentagem dos tumores benignos do membro superior, contudo é o mais frequente de origem neural. Apresentamos um estudo retrospectivo de schwanomas do membro superior para estabelecer a abordagem pré-operatória adequada, avaliar a eficácia do tratamento e observar a evolução pós-operatória.

Material e Métodos: Revisão de 17 doentes tratados desde 2007 a 2014 com schwanoma do membro superior e caracterização quanto à idade, sexo, localização no membro superior, sinais e sintomas, exames pré-operatórios, diagnóstico histológico e vigilância pós-operatória.

Resultados: Observou-se que em quatro dos doentes, o schwanoma se localizava no braço, três no antebraço, quatro no punho e seis na mão ou dedos. Procedeu-se a excisão com ampliação óptica para optimização do resultado funcional final. A enucleação foi realizada sem lesão fascicular em 12 doentes. Por sua vez, em cinco doentes efectuou-se a excisão dos fascículos envolvidos pela massa. No pós-operatório imediato as parestesias surgiram em nove doentes, regredindo em cinco daqueles no período pósoperatório de 12 meses. Um doente evidenciou défice motor do nervo radial, com recuperação parcial aos 12 meses.

Discussão: Os schwanomas são tumores raros, de morfologia bem delimitada, que atingem esporadicamente o membro superior. $O$ diagnóstico pré operatório é pouco frequente, pelo que normalmente apenas se estabelece o diagnóstico após a excisão e o estudo histopatológico.

Conclusão: Os schwanomas deverão ser equacionados nas hipóteses de diagnóstico dos nódulos subcutâneos associados a sintomas neurológicos. Assim, é importante investigar a presença de parestesias e o sinal de Tinel, característicos desta lesão. A imagiologia tem um contributo fruste no diagnóstico. Para melhorar o resultado final, o estudo pré-operatório deverá ser criterioso, sendo aconselhável ponderar o benefício da cirurgia face à potencial lesão iatrogénica do nervo.

Palavras-chave: Extremidade Superior; Mão; Neoplasias de Tecidos Moles; Neurilemoma.

\section{ABSTRACT}

Introduction: Schwannoma or neurilemmoma is a benign peripheric nerve tumor that usually presents as a slow growing single lesion; it has origin in Schwann cells proliferation. Although it represents a small percentage of the benign tumors of the upper arm, it is the most frequent of neural origin. We present a retrospective study of upper limb schwannomas; our aim is establish the appropriate preoperative approach, to recognise the efficiency of the treatment and the pos-operative follow-up.

Material and Methods: Review of 17 patients treated between 2007 and 2014 with upper limb schwannoma and characterization as to age, gender, location in the upper limb, signs and symptoms, pre-operative studies, histologic diagnosis and postoperative surveillance. Results: In four of them the schwannoma was localized on the arm, three on the forearm, four on the wrist and 6 on the hand or fingers. We proceed to careful microsurgical dissection in a bloodless field to optimize the functional final result. The enucleation without fascicular lesion was achieved in 12 patients. In five patients we performed the excision of the fascicles involved by the mass. Nine patients developed paresthesias in the immediate postoperative period, with five of them improving in 12 months. One patient presented motor deficit of the radial nerve with partial recover at 12 monts.

Discussion: Schwannomas are rare tumours with well circumscribed morphology. Occasionally it appears in upper limb. The preoperative diagnosis is rarely, so normally the diagnosis is established only after excision and histologic study.

Conclusion: Schwannoma should be considered as a diagnostic hypothesis when evaluating subcutaneous nodes associated with neurologic symptoms. It is important to query the presence of paresthesias and perform the Tinel sign, both typical of this condition. Imagiological exams have a scarce contribute in the diagnosis. To improve the final result, the preoperative study must be insightful, being advisable to consider the benefits of the surgery compared to the potential iatrogenic damage to the nerve.

Keywords: Hand; Neurilemmoma; Soft Tissue Neoplasms; Upper Extremity.

\section{INTRODUÇÃO}

O schwanoma, designado anteriormente por neurilemoma, é um tumor benigno da bainha dos nervos periféricos com origem na proliferação das células de Schwann. Manifesta-se habitualmente como lesão única de crescimento lento e indolor ao longo de vários anos, sem sintomas específicos. ${ }^{1,2}$ Contudo, por vezes surge num contexto de

múltiplas lesões, descrito na literatura em raros casos não associados a síndromes familiares. ${ }^{3-5}$

Os schwanomas esporádicos manifestam-se sobretudo entre a terceira e sexta década sem predomínio de género. ${ }^{1}$ Surgem em qualquer localização, com a cabeça e pescoço a serem as regiões mais frequentemente afectadas,

1. Serviço de Cirurgia Plástica, Reconstrutiva e Maxilo-Facial. Hospital Egas Moniz. Centro Hospitalar de Lisboa Ocidental. Lisboa. Portugal.

$\bowtie$ Autor correspondente: Ângelo Sá. jose.angelo.sa@gmail.com

Recebido: 11 de agosto de 2015 - Aceite: 16 de fevereiro de 2016 | Copyright $\odot$ Ordem dos Médicos 2016 
seguidas do tronco, membro inferior e, finalmente, superior. Apesar de serem raros e corresponderem a menos de $5 \%$ - $8 \%$ de todos os tumores do membro superior, ${ }^{1,6,7}$ são os tumores benignos de origem neural mais frequentes neste segmento, seguidos dos neurofibromas. , $^{1,2,6,7}$ Manifestam-se sobretudo na região flexora, devido à elevada concentração de estruturas nervosas neste compartimento. ${ }^{6,7} \mathrm{Na}$ mão e punho são raros, com uma taxa de $1 \%$ dos schwanomas reportados. ${ }^{7}$

O propósito deste estudo retrospectivo de 17 casos de schwanomas do membro superior, tratados num intervalo de oito anos, foi definir o correcto estudo pré-operatório, avaliar o tratamento cirúrgico, assim como os défices neurológicos a curto e longo prazo.

\section{MATERIAL E MÉTODOS}

Entre janeiro de 2007 e dezembro de 2014, foram operados no Centro Hospitalar de Lisboa Ocidental 17 doentes com schwanoma no membro superior. Apenas foram incluídos aqueles com diagnóstico histopatológico definitivo de schwanoma no membro superior após a excisão completa. Os dados foram adquiridos a partir dos registos clínicos pré e pós-operatórios. Foram ainda consultados os relatórios imagiológicos e histopatológicos.

Os parâmetros avaliados foram a idade, sexo, localização no membro superior, sinais e sintomas, exames pré-operatórios, diagnóstico histológico e vigilância pós-operatória (Tabela 1).

O grupo era composto por cinco doentes do sexo mas- culino e 12 do sexo feminino; a média de idades foi 60,2 anos, num intervalo de 25 - 85 anos de idade. No que se refere à localização, seis foram encontrados no nervo mediano ou ramos digitais, quatro identificados no nervo radial ou ramos terminais, dois localizados no nervo cubital, dois encontrados no nervo cutâneo medial do braço e três no nervo cutâneo medial do antebraço. Topograficamente, quatro schwanomas estavam localizados no braço, três no antebraço, quatro no punho e seis na mão ou dedos. Constatou-se que 10 dos 17 doentes (59\%) apresentavam lesão da bainha dos nervos na mão e punho. A tumefacção palpável foi encontrada em 12 casos (71\%). As dimensões dos tumores variaram entre $4 \mathrm{~mm}$ e $7 \mathrm{~cm}$ de diâmetro. Os sintomas predominantes foram dor e parestesias, com o primeiro, frequentemente de origem súbita, a estar presente em 11 dos casos (65\%) e, o último, observado em 13 dos 17 doentes (76\%). O sinal de Tinel, que é positivo se se desencadear parestesias no território do nervo afectado após a percussão sobre a massa, foi objectivado em 14 doentes (82\%) (Tabela 2).

No estudo pré-operatório cinco doentes foram estudados por ecografia, enquanto quatro doentes realizaram ressonância magnética (RM). Nos restantes doentes não se recorreu a exames auxiliares de diagnóstico. A biópsia diagnóstica não foi efectuada em nenhum dos casos, tendo sido preterida pela exploração cirúrgica.

Todos os doentes foram tratados com excisão por meio de ampliação óptica com lupas. Procedeu-se a incisão longitunal do epinervo sobre o tumor e enucleação de

Tabela 1 - Caracterização dos doentes

\begin{tabular}{|c|c|c|c|c|c|}
\hline Caso & Idade / Sexo & Localização & Nervo & Imagiologia & Dimensões (cm) \\
\hline 1 & 34 우 & DI & $\mathrm{N}$ colateral mediano & - & $1,0 \times 0,8$ \\
\hline 2 & 33 우 & Braço & $\mathrm{N}$ cutâneo medial do braço & - & $1,5 \times 1,7$ \\
\hline 3 & $25+$ & Antebraço & $\mathrm{N}$ radial & - & $3,0 \times 1,2$ \\
\hline 4 & $65+$ & Mão & Ramo terminal do $\mathrm{N}$ radial & - & $0,5 \times 1,0$ \\
\hline 5 & 63 ㅇ & Antebraço & $\mathrm{N}$ cutâneo medial do antebraço & - & $1,7 \times 0,7$ \\
\hline 6 & 73 안 & Mão & $\mathrm{N}$ colateral mediano & Ecografia & $1,1 \times 0,9$ \\
\hline 7 & $71 \hat{\jmath}$ & Braço & $\mathrm{N}$ radial & RM & $7,0 \times 0,8$ \\
\hline 8 & $53+$ & Punho & $\mathrm{N}$ cubital & RM & $1,8 \times 1,5$ \\
\hline 9 & $73 \hat{\jmath}$ & Mão & $\mathrm{N}$ colateral mediano & Ecografia & $1,0 \times 0,6$ \\
\hline 10 & $75 \lesssim$ & Punho & Ramo terminal do $\mathrm{N}$ radial & Ecografia & $0,4 \times 0,5$ \\
\hline 11 & 48 ㅇ & Antebraço & $\mathrm{N}$ cutâneo medial do antebraço & - & $1,1 \times 0,8$ \\
\hline 12 & $72 \hat{\sigma}$ & Braço & $\mathrm{N}$ cutâneo medial do braço & - & $1,0 \times 0,8$ \\
\hline 13 & 58 운 & DIII & $\mathrm{N}$ colateral mediano & - & $1,5 \times 0,5$ \\
\hline 14 & 46 ㅇ & DI & $\mathrm{N}$ colateral mediano & Ecografia & $0,7 \times 0,5$ \\
\hline 15 & 78 우 & Punho & $\mathrm{N}$ cubital & RM & $1,7 \times 1,2$ \\
\hline 16 & $71+$ & Punho & $\mathrm{N}$ mediano & RM & $1,0 \times 0,6$ \\
\hline 17 & $85 \hat{\jmath}$ & Braço & $\mathrm{N}$ cutâneo medial do antebraço & - & $1,0 \times 0,9$ \\
\hline
\end{tabular}


neoformação em 12 doentes, sem lesão fascicular adicional, para além do fascículo já envolvido, decorrente da dissecção interfascicular minuciosa. Nos restantes foram ressecados os fascículos envolvidos pela massa.

No período de vigilância realizou-se consulta de avaliação nas duas primeiras semanas e subsequentemente aos três, seis e 12 meses. Os sintomas avaliados foram parestesias e dor.

Os autores declaram que o estudo foi aprovado pela Comissão de Ética de acordo com a Declaração de Helsínquia.

\section{RESULTADOS}

A histologia das lesões excisadas demonstrou aspectos compatíveis com schwanomas em 17 casos. O diagnóstico pré-operatório foi efectuado em cinco dos casos, tendo sido adiantados outros diagnósticos como neurofibroma, lipoma ou tumor de células gigantes. A ecografia foi realizada em cinco casos, com três a estabelecer o diagnóstico pré-operatório correcto (Fig. 1). Por sua vez, a RM estabeleceu o diagnóstico correcto em dois dos quatro doentes que realizaram o exame.

No grupo daqueles em que se realizou a enucleação, cinco doentes evidenciaram complicações neurológicas no pós-operatório imediato, traduzindo-se apenas em alterações da sensibilidade. Não se registaram outras complicações. No grupo em que se efectuou a ressecção de fascículos incluídos na massa, todos os casos apresentaram complicações precoces, com parestesias em quatro casos e défice motor num quinto doente, sinal que não se detectava no pré-operatório.

Os défices sensitivos foram tolerados e regrediram até aos 12 meses após a cirurgia em cinco doentes, com persistência em quatro, um pertencente ao grupo daqueles em que se realizou enucleação. Além disso, um doente exibiu aos 12 meses défice motor parcial. A dor e a presença de sinal de Tinel evoluíram com remissão completa.

A localização do tumor e o nervo afectado não parecem modificar o resultado final. A excepção aplica-se aos schwanomas dos nervos digitais que se caracterizam invariavelmente por défice sensitivo no pós- operatório.

A completa enucleação do schwanoma com a preservação dos fascículos ou a inclusão de outros fascículos além da origem tumoral na excisão não afectou o resultado no pós-operatório de forma significativa.

Não se registou nenhuma recidiva ou atrofia muscular no período de vigilância.

\section{DISCUSSÃO}

Os tumores dos nervos periféricos são raros, podendo ser benignos ou malignos. O schwanoma inclui-se nos primeiros e apresenta crescimento lento e excêntrico, sem lesão dos fascículos nervosos (Fig. 2).

Trata-se de uma lesão capsulada com morfologia bem delimitada, pelo que pode ser excisado com poupança do nervo (Fig. 3). $\cdot^{7-10}$ Porém, uma parte dos schwanomas

Tabela 2 - Sinais e sintomas associados a schwanomas do membro superior

\begin{tabular}{|c|c|c|c|c|c|c|c|}
\hline \multirow[b]{2}{*}{ Caso } & \multirow[b]{2}{*}{ Localização } & \multirow[b]{2}{*}{ Nervo } & \multicolumn{3}{|c|}{ Pré-operatório } & \multicolumn{2}{|c|}{ Pós-operatório } \\
\hline & & & Dor & Parestesias & Sinal Tinel & $15^{\circ}$ dia PO & $12^{\circ}$ mês PO \\
\hline 1 & DI & Mediano & $x$ & $x$ & $x$ & Parestesias & Sem queixas \\
\hline 2 & Braço & $\mathrm{CMB}^{*}$ & & & $x$ & \multicolumn{2}{|c|}{ Sem queixas } \\
\hline 3 & Antebraço & Radial & & $x$ & $x$ & Parestesias & Sem queixas \\
\hline 4 & Mão & Radial & $x$ & $x$ & $x$ & Parestesias & Sem queixas \\
\hline 5 & Antebraço & $\mathrm{CMA} \dagger$ & & $x$ & $x$ & \multicolumn{2}{|c|}{ Sem queixas } \\
\hline 6 & DIII & Mediano & $x$ & & $x$ & Parestesias & Sem queixas \\
\hline 7 & Braço & Radial & $x$ & $x$ & $x$ & Défice motor & Remissão parcial \\
\hline 8 & Punho & Cubital & $x$ & $x$ & $x$ & Parestesias & Sem queixas \\
\hline 9 & DII & Mediano & $x$ & $x$ & & Parestesias & Parestesias \\
\hline 10 & Punho & Radial & $x$ & $x$ & $x$ & Parestesias & Sem queixas \\
\hline 11 & Antebraço & $\mathrm{CMA} \dagger$ & & & $x$ & \multicolumn{2}{|c|}{ Sem queixas } \\
\hline 12 & Braço & $\mathrm{CMB}^{*}$ & & $x$ & $x$ & \multicolumn{2}{|c|}{ Sem queixas } \\
\hline 13 & DIII & Mediano & $x$ & $x$ & & Parestesias & Sem queixas \\
\hline 14 & $\mathrm{DI}$ & Mediano & $x$ & $x$ & & Parestesias & Parestesias \\
\hline 15 & Punho & Cubital & $x$ & $x$ & $x$ & \multicolumn{2}{|c|}{ Sem queixas } \\
\hline 16 & Punho & Mediano & $x$ & $x$ & $x$ & \multicolumn{2}{|c|}{ Semqueixas } \\
\hline 17 & Braço & $\mathrm{CMA} \dagger$ & & & $x$ & \multicolumn{2}{|c|}{ Sem queixas } \\
\hline
\end{tabular}

* Nervo cutâneo medial braquial. † Nervo cutâneo medial antebraquial. 


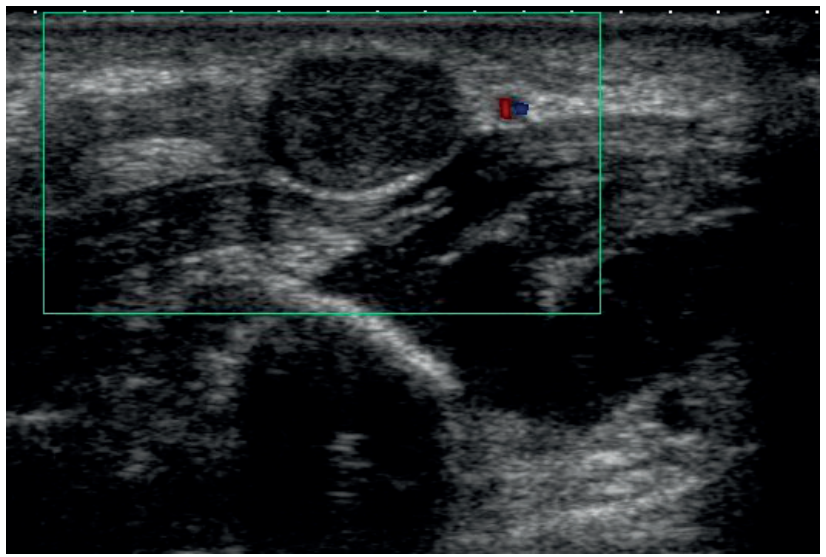

Figura 1 - Imagem ecográfica de lesão schwanomatosa

apresenta envolvimento de vários fascículos nervosos, o que poderá motivar lesão neurológica aquando da excisão por impossibilidade de os preservar, como observado em vários estudos..$^{11-13}$ No estudo efectuado, onde se procedeu apenas à análise dos casos de Schwanoma do membro superior pela apetência do serviço para este segmento anatómico, verificamos parestesias no pós-operatório imediato com taxa de incidência de 53\%.

Os schwanomas onde não é possível isolar os fascículos nervosos apresentam comportamento idêntico aos neurofibromas. Estes últimos definem-se por crescimento intraneural e dano fascicular, não sendo possível proceder à enucleação e preservar a função sensitiva ou motora, com o défice final a ser sobreponível. . $^{13,14}$ No presente estudo, a dissecção fascicular não foi possível em cinco doentes, os quais surgiram no pós-operatório com alterações neurológicas, que persistiram em três deles aos 12 meses.

Clinicamente, numa fase inicial são silenciosos, contudo evoluem com dor e parestesias decorrentes do efeito de massa local. $2,5,8$ Assim, os sintomas não dependem das dimensões do tumor, mas do grau de compressão e ausência de distensibilidade tecidular local. ${ }^{2,8} \mathrm{Na}$ mão e punho as queixas de dor são atribuíveis à reduzida expansibilidade das estruturas locais. ${ }^{6,12,15}$

No estudo realizado, 10 doentes possuíam a lesão neural localizada na mão ou punho, justificando-se a maior inci-

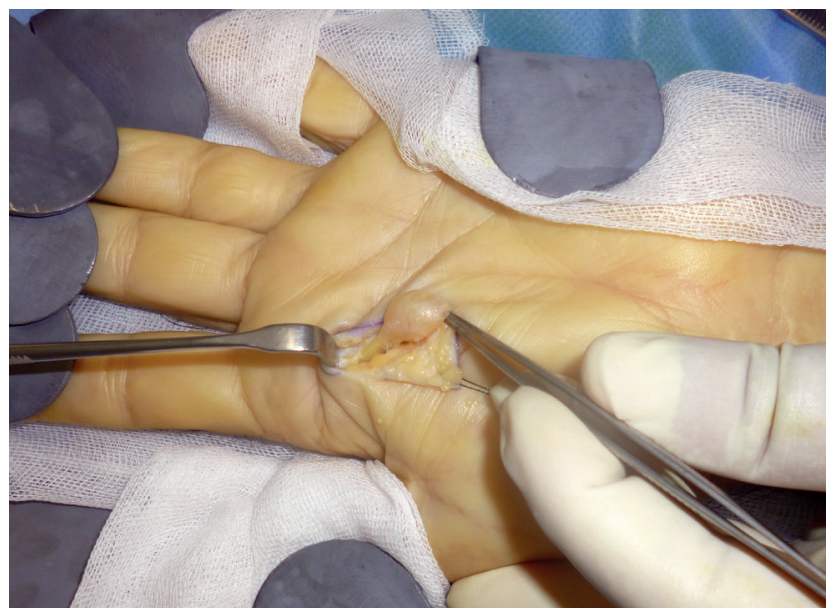

Figura 2 - Neoformação do nervo de crescimento excêntrico dência na extremidade do membro superior, pelo facto das estruturas nervosas serem mais numerosas distalmente. ${ }^{11}$

O schwanoma nos dedos foi encontrado em cinco doentes, evidenciando-se a dor como o sintoma transversal a todos.

O diagnóstico pré-operatório de schwanoma com base na história clínica e exame físico é dificilmente realizado devido à baixa incidência, bem como pelo facto de outras lesões de origem neural mimetizarem os sintomas. ${ }^{15,9,15} \mathrm{No}$ exame objectivo pode detectar-se uma neoformação subcutânea ao longo do trajecto nervoso, móvel transversal, mas não longitudinalmente. ${ }^{2,11}$ Tal como reportado por outros autores, ${ }^{1,8,14,15}$ o sinal de Tinel sobre a massa, que foi objectivado em $82 \%$ dos nossos doentes, está frequentemente presente.

Na revisão efectuada constatou-se a realização de ecografia em cinco casos e RM em quatro doentes. Os exames imagiológicos nem sempre são conclusivos, dado a semelhança destes com os neurofibromas..$^{1,5,8}$ Nos exames sugestivos de schwanoma não é possível definir o envolvimento fascicular, bem como se a massa poderá ser completamente enucleada. ${ }^{11}$ Todavia, apesar de por vezes ser difícil estabelecer o diagnóstico apenas com os exames imagiológicos, estes têm um papel importante na determinação das relações anatómicas da massa com as estruturas circundantes. ${ }^{2}$

O exame histológico é o único que certifica o diagnóstico, ${ }^{9}$ no entanto a biópsia é desaconselhada quando há suspeita de lesão da bainha do nervo periférico, pelo risco de lesão iatrogénica permanente do nervo, bem como a fibrose local que dificultará a dissecção delicada na excisão. ${ }^{14}$ Deste modo, quando perante a suspeita de schwanoma deverá ser explorado cirurgicamente e excisado, com a explicação prévia ao doente da possibilidade de lesão nervosa e défices neurológicos subjacentes. ${ }^{14}$

O tratamento de eleição dos schwanomas é a enucleação do tumor do nervo envolvido sob ampliação óptica. $5,8,10$ A técnica utilizada poderá ser microdissecção intracapsular ou extracapsular. ${ }^{14} \mathrm{~A}$ primeira, a abordagem utilizada no grupo de doentes deste estudo por preferência dos cirurgiões, apresenta menor risco de lesão iatrogénica.

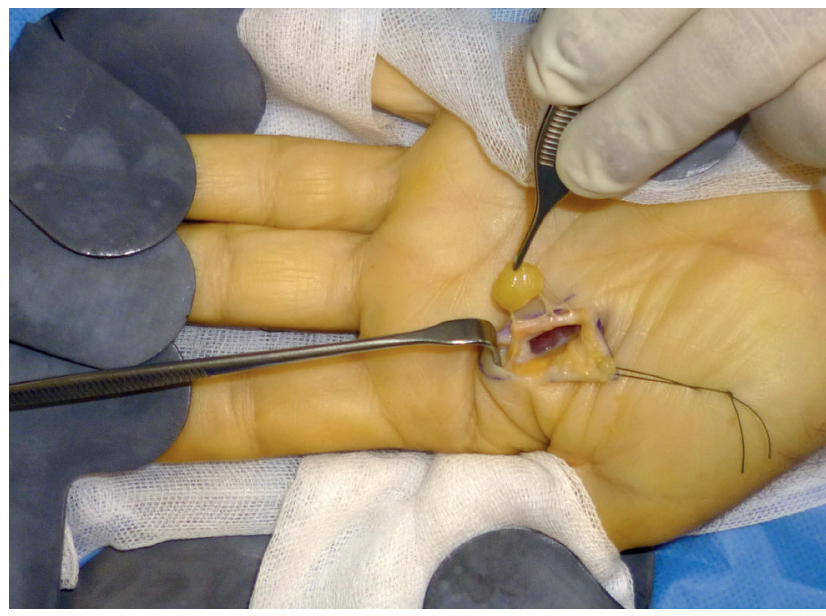

Figura 3 - Dissecção com preservação dos fascículos nervosos 


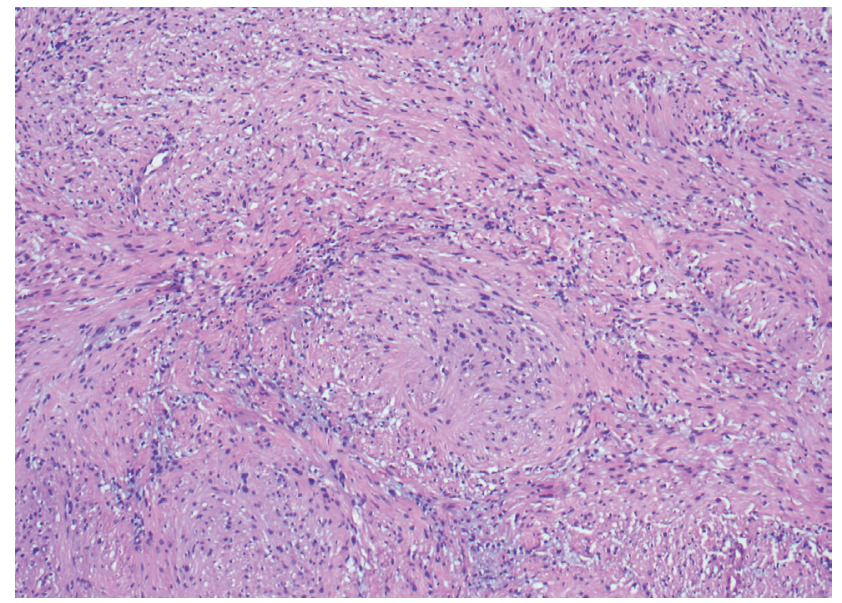

Figura 4 - Estudo microscópico do schwanoma (ampliação 4x)

Acreditamos que a dissecção intracapsular é mais fácil de executar, tal como mais segura. A atenção deverá ser dirigida para os pólos proximal e distal, onde os fascículos poderão não ser facilmente destacáveis do schwanoma. ${ }^{14}$ Contudo, a enucleação do tumor sem qualquer lesão fascicular apenas ocorreu em 12 doentes dos 17 tratados. Nos restantes casos procedeu-se à ressecção dos fascículos indissociáveis. A reparação não foi realizada por percepção de ausência de benefício final.

Macroscopicamente os schwanomas são capsulados com superfície lisa, por vezes com o nervo visível, coloração branca ou amarelada e focos hemorrágicos e/ou císticos. ${ }^{9,15}$ Microscopicamente são bem delimitados e demostram arquitectura bifásica de padrão de Antoni A com células fusiformes e núcleos em paliçada (imunopositividade para a proteína S100) e B com menor celularidade. ${ }^{6,9,15}$ (Fig. 4)

Este tumor tem um excelente prognóstico com baixa taxa de recorrência e malignização, com a excisão curativa a ser bastante eficaz. ${ }^{5,7,15}$ No presente estudo não se comprovou nenhuma recidiva.

Apesar de todo o cuidado na dissecção, um número elevado de doentes referiu distúrbios sensitivos e/ou motores no pós-operatório, como é largamente relatado na literatura. ${ }^{13,14} \mathrm{O}$ limiar de lesão iatrogénica na dissecção cirúrgica de um nervo periférico é mais baixo na extremidade superior do que no tronco ou membros inferiores. ${ }^{14}$

No nosso estudo, reportamos parestesias no pós-operatório em $53 \%$ dos doentes. As causas subjacentes foram enumeradas por Sawada et a/13: primeiro o schwanoma tem origem na bainha do nervo e contém um fascículo que está sempre envolvido independemente da função residual que desempenha, pelo que a secção origina défice neurológico adicional; a incisão longitudinal aquando da dissecção pode seccionar alguns fascículos; e os restantes fascículos preservados são sujeitos a compressão e retracção durante a excisão, o que motiva neuropraxia no pós-operatório imediato. Donner et a/12 demonstraram que os fascículos envolvidos na lesão e, que foram obrigatoriamente seccionados, já apresentavam função residual na transmissão dos estímulos nervosos. Deste modo, defendem que a secção não é motivo suficiente para desencadear o quadro persistente de parestesias. Por outro lado, em dois recentes estudos, Park et $a /^{14}$ e Tang et a/11 sustentam opinião diferente, atribuindo as parestesias no pós-operatório imediato à secção dos fascículos na dissecção. Neste estudo comprovamos que, onde a dissecção tumoral não poupou os fascículos adjacentes à massa, os doentes apresentaram no pós-operatório maiores queixas de parestesias. Assim, acreditamos que a lesão fascicular é a razão primordial para o quadro persistente de parestesias.

$O$ resultado final do presente estudo apresenta resultados relativamente sobreponíveis a outros relatos..$^{1,13,14}$

\section{CONCLUSÃO}

Os schwanomas são lesões benignas raras da bainha dos nervos periféricos que devem integrar as hipóteses de diagnóstico nas tumefacções associadas a sintomas neurológicos do membro superior. Para melhorar o resultado final, o estudo pré-operatório necessita de ser criterioso e se houver suspeita de schwanoma, o doente deve ser informado do elevado risco de défice neurológico temporário ou permanente no pós-operatório. Deste modo, o risco e benefício da excisão devem ser ponderados, com o doente a estar envolvido na decisão. Embora se realize dissecção microcirúrgica com enucleação intracapsular, o risco de défice temporário ou definitivo não está excluído.

\section{PROTECÇÃO DE PESSOAS E ANIMAIS}

Os autores declaram que os procedimentos seguidos estavam de acordo com os regulamentos estabelecidos pelos responsáveis da Comissão de Investigação Clínica e Ética e de acordo com a Declaração de Helsínquia da Associação Médica Mundial.

\section{CONFIDENCIALIDADE DOS DADOS}

Os autores declaram ter seguido os protocolos do seu centro de trabalho acerca da publicação de dados.

\section{CONFLITOS DE INTERESSE}

Os autores declaram não terem qualquer conflito de interesse relativamente ao presente artigo.

\section{FONTES DE FINANCIAMENTO}

Os autores declaram não ter recebido subsídios ou bolsas para a elaboração do artigo.

\section{REFERÊNCIAS}

1. Ozdemir O, Ozsoy MH, Kurt C, Coskunol E, Calli I. Schwannomas of the hand and wrist: long-term results and review of the literature. J Orthop Surg. 2005;13:267-72.

2. Sandberg K, Nilsson J, Søe Nielsen N, Dahlin LB. Tumours of peripheral

nerves in the upper extremity: a 22-year epidemiological study. Scand J Plast Reconstr Surg Hand Surg. 2009;43:43-9.

3. Patel MR, Mody K, Moradia VJ. Multiple schwannomas of the ulnar nerve: a case report. J Hand Surg Am. 1996;21:875-6. 
4. Tanabe K, Tada K, Ninomiya H. Multiple schwannomas in the radial nerve. J Hand Surg Br. 1997;22:664-6.

5. Kütahya H, Güleç A, Güzel Y, Kacira B, Toker S. Schwannoma of the median nerve at the wrist and palmar regions of the hand: a rare case report. Case Rep Orthop. 2013;2013:950106.

6. Hasham S, Matteucci P, Stanley PR. Schwannomatosis: multiple schwannomas of the upper limb. J Hand Surg Br. 2006;31:182-4.

7. Rockwell GM, Thoma A, Salama S. Schwannoma of the hand and wrist. Plast Reconstr Surg. 2003;111:1227-32.

8. Adani R, Tarallo L, Mugnai R, Colopi S. Schwannomas of the upper extremity: analysis of 34 cases. Acta Neurochir. 2014;156:2325-30.

9. Adani R, Baccarani A, Guidi E, Tarallo L. Schwannomas of the upper extremity: diagnosis and treatment. Chir Organi Mov. 2008;92:85-8.

10. Aslam N, Kerr G. Multiple schwannomas of the median nerve: a case report and literature review. Hand Surg. 2003;8:249-52.

11. Tang CY, Fung B, Fok M, Zhu J. Schwannoma in the upper limbs. Biomed Res Int. 2013;2013:16796.

12. Donner TR, Voorhies RM, Kline DG. Neural sheath tumors of major nerves. J Neurosurg. 1994;81:362-73.

13. Sawada T, Sano M, Ogihara H, Omura T, Miura K, Nagano A. The relationship between pre-operative symptoms, operative findings and postoperative complications in schwannomas. J Hand Surg $\mathrm{Br}$. 2006;31:629-34.

14. Park MJ, Seo KN, Kang HJ. Neurological deficit after surgical enucleation of schwannomas of the upper limb. J Bone Joint Surg Br. 2009;91:1482 6.

15. Hubert J, Landes G, Tardif M. Schwannoma of the median nerve. J Plast Surg Hand Surg. 2013;47:75-7. 